\title{
Comparison of two different coagulation algorithms on the use of allogenic blood products and coagulation factors in severely injured trauma patients: a retrospective, multicentre, observational study
}

Alexander Kaserer $^{1 *}$ (D), Mattias Casutt ${ }^{2}$, Kai Sprengel ${ }^{3}$, Burkhardt Seifert ${ }^{4}$, Donat R. Spahn ${ }^{1}$ and Philipp Stein ${ }^{1}$

\begin{abstract}
Background: At the University Hospital Zurich (USZ) and the Cantonal Hospital of Lucerne (LUKS) an individualized goal-directed coagulation and transfusion algorithm was introduced and implemented before 2012 (Coagulation algorithm of the USZ: USZ-Alg; of the LUKS: LUKS-Alg). Main differences between both algorithms are: 1) A target haematocrit-range of 0.21-0.24 (USZ-Alg) vs. a lower haematocrit limit only (LUKS-Alg). 2) Blind coagulation-package in selected cases (LUKS-Alg only). 3) Factor XIII substitution is considered earlier according to the USZ-Alg. The Aim of this study was to analyse the impact of two different coagulation algorithms on the administration of allogeneic blood products, coagulation factors, the frequency of point of care measurements and haemoglobin level during resuscitation in trauma patients.

Methods: This retrospective, multicentre, observational study included all adult trauma patients with an injury severity score (ISS) $\geq 16$ primarily admitted to the USZ or the LUKS in the period of 2012 to 2014. Referred patients and patients with missing/incomplete records of the initial treatment at the emergency department (ED) were excluded. Two propensity score matched groups were created using a non-parsimonious logistic regression to account for potential differences in patient and trauma epidemiology.

Results: A total of 632 patients meeting the inclusion criteria were admitted to the two hospitals: 428 to the USZ and 204 to the LUKS. Two Propensity score matched groups ( $n=172$ per group) were created. Treatment with USZ-Alg compared with LUKS-Alg resulted in a lower number of patients receiving RBC transfusion (11.6\% vs. 29.7\%, OR 3.2, 95\% Cl 1.8-5.7, $p<0.001)$ and lower amount of RBC transfusion (0.5 SD 1.9 vs. 1.5 SD 3.9, $p<0.001)$. The different treatment algorithms resulted in lower mean haemoglobin levels in the USZ during resuscitation (8.0 SD 1.7 vs. $9.4 \mathrm{SD} 1.8 \mathrm{~g} / \mathrm{dl}, p<0.001$ ) and at admission to the ICU (8.3 SD $1.2 \mathrm{vs.} 10.6 \mathrm{SD} 1.9 \mathrm{~g} / \mathrm{dl}, p<0.001$. Blood gas analyses to monitor treatment and haematocrit were made more frequently in the USZ (1.4 SD 0.8 vs. 1.0 SD 0.7 measurements per hour, $p=0.004$ ).

(Continued on next page)
\end{abstract}

\footnotetext{
*Correspondence: Alexander.Kaserer@usz.ch

${ }^{1}$ Institute of Anaesthesiology, University and University Hospital Zürich,

Raemistrasse 100, 8091 Zürich, Switzerland

Full list of author information is available at the end of the article
} 
(Continued from previous page)

Conclusion: A goal-directed coagulation algorithm including a target haematocrit-range including frequent and repeated haematocrit measurement may lead to less transfusion of RBC compared to only a lower haematocrit limit, when treating severely traumatized patients.

Keywords: Coagulation algorithm, Coagulation management, Point of care measurements, Transfusion, Trauma

\section{Background}

Individualized goal-directed point-of-care and factor concentrate based coagulation and transfusion algorithms to treat massively bleeding patients following severe trauma have been published [1-4]. Such algorithms require adequate, fast and goal-directed replacement of plasma components and coagulation factors to tackle trauma induced coagulopathy and its consequences, particularly mortality $[5,6]$. It could already be shown that the implementation of transfusion and coagulation algorithms leads to an improved outcome with a significant reduction of mortality, massive transfusion and the use of allogeneic blood products $[1,3,7]$. Coagulation management according to individualized goal-directed coagulation and transfusion algorithms is one of the key elements in the European Trauma Treatment Guidelines [8].

In 2008, the University Hospital of Zurich (USZ), Switzerland, implemented an algorithm for goal directed transfusion and coagulation management based on the pathophysiology of a developing coagulopathy in massively bleeding patients [9]. The algorithm was subsequently revised and then fully implemented in 2012 [1, 10, 11]. A similar algorithm was implemented in the Cantonal Hospital of Lucerne (LUKS), Switzerland, in 2011.

Although both algorithm base on the same principles and are largely comparable, some differences exist. These differences may have significant influence on the administration of allogeneic blood products and coagulation factors. To the best of our knowledge no study has been conducted and published comparing two goaldirected, point of care and factor based coagulation algorithms. The aim of this study was to analyse the impact of the differences between two distinct transfusion and coagulation algorithms on the administration of allogeneic blood products, coagulation factors, the frequency of point of care measurements and haemoglobin levels during resuscitation.

\section{Methods}

This study was approved by the local ethics committee (Kantonale Ethikkommission Zürich, Switzerland, KEK $\mathrm{ZH}$ 2015-0309) and adheres to the Strengthening the Reporting of Observational Studies in Epidemiology (STROBE) recommendations for cohort studies [12].

\section{Study design and participants}

This retrospective, multicentre, observational study includes all adult trauma patients ( $\geq 16$ years) with an Injury Severity Score (ISS) $\geq 16$ primarily admitted to the USZ or the LUKS between January 1, 2012 and December 31, 2014.

Exclusion criteria were secondary transfer to the USZ or LUKS, Age $<16$ years and missing/incomplete records of the initial treatment at the emergency department (Fig. 1).

\section{Setting}

The USZ and LUKS are two of twelve level 1 trauma centres in Switzerland. All severely injured patients are transferred by ambulance or helicopter to one of these level 1 trauma centres. A standardized clinical approach is provided in the emergency department (ED) of the USZ and LUKS consisting of a primary survey and further treatment according to $\mathrm{ATLS}^{\circ}$ or $\mathrm{ETC}^{\circ}$. The trauma staff at the ED contains at least one senior and one junior anaesthetist one senior and one junior trauma surgeon. An initial whole-body CT scan is performed in all

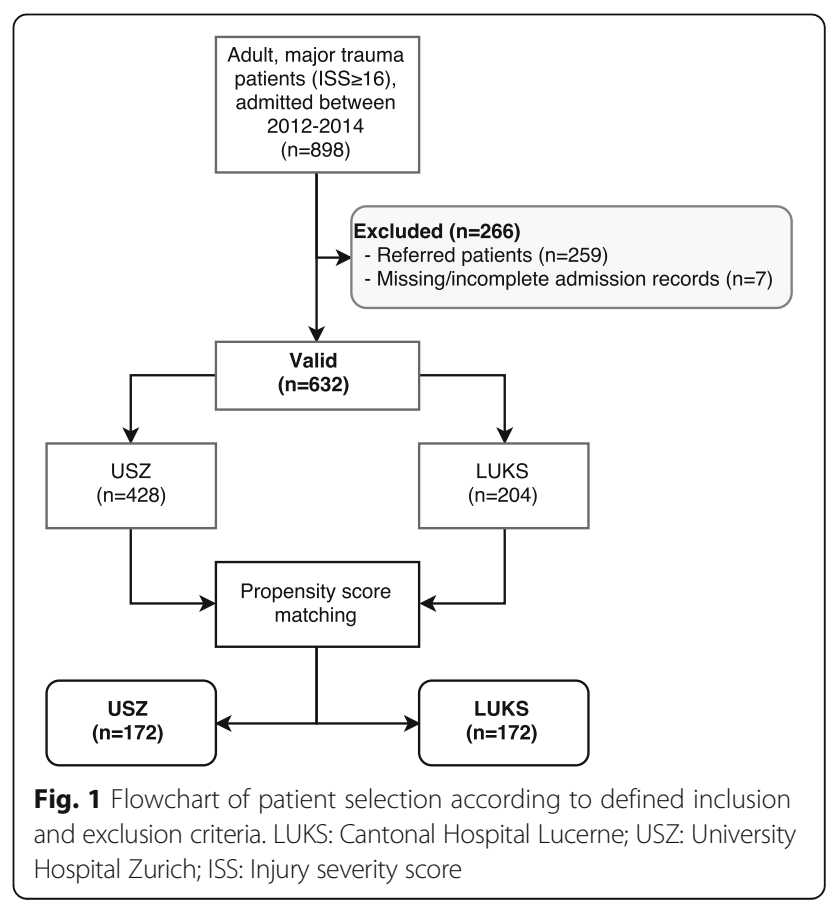


major trauma patients as soon as possible to evaluate their relevant injuries and to determine further treatment [13].

\section{Transfusion and coagulation algorithm}

At the USZ in 2008 an individualized goal-directed transfusion and coagulation algorithm was introduced, revised and fully implemented until 2012 (USZ-Alg) [9-11]. At the LUKS, a similar coagulation algorithm was introduced and implemented during 2011 (LUKS-Alg). Both algorithm guide the transfusion and coagulation management for all massively bleeding trauma patients.

\section{Basics of both algorithm}

On admission to the ED, blood samples to determine haemoglobin level, platelet count, fibrinogen level, factor $\mathrm{V}$ and XIII activity, international normalized ratio (INR), aPTT, blood gas analysis (ABGA) and ROTEM ${ }^{\odot}$ (Rotational thromboelastometry, TEM International, Munich, Germany) analysis are taken. Haematology assays and factor activity measurement are available $24 \mathrm{~h}$ a day, within 30 min. ROTEM $^{\circledR}$ measurements include EXTEM (tissue factor activated extrinsic pathway), INTEM (ellagic acid activated intrinsic pathway), FIBTEM (containing platelet inhibitor cytochalasin $\mathrm{D}$, evaluating the contribution of fibrinogen to clot formation) and APTEM (containing aprotinin to inhibit plasmin to evaluate fibrinolysis). The first step is to screen patient's medical history and medication to identify factors affecting coagulation (antiplatelet drugs, heparin, oral anticoagulants or history of immune reactions altering coagulation). General target values include normothermia, normocalcemia, normal acid-base status, adequate haematocrit, permissive hypotension (mean arterial pressure $55-60 \mathrm{mmHg}$ ). Fluid resuscitation should primarily be performed with balanced crystalloid solutions. If colloids are considered to be useful, only gelatin administration is recommended in both algorithms. Tranexamic acid (TXA), fibrinogen concentrate, 4 factor prothrombin complex concentrate (PCC), factor XIII and allogeneic blood products are administered according to laboratory, ABGA and ROTEM ${ }^{\bullet}$ findings. If target values are reached, diffuse bleeding continues and a DIC is not likely, recombinant factor VIIa may be evaluated.

\section{Main differences between both algorithm}

- For the RBC transfusion management, a target haematocrit-range of $0.21-0.24$ is specified in USZ-Alg, whereas in LUKS-Alg only a lower haematocrit limit of 0.21 is defined.

- LUKS-Alg contains the option for selected Trauma patients (age $<50$ years, no fatal injury, temperature $>35^{\circ} \mathrm{C}$ and diffuse bleeding) to administer the following transfusion - and coagulation package blindly and not goal-directed: 2 g Tranexamic acid, 4 g Fibrinogen, 1'000 U Prothrombin complex concentrate, 4 RBCs (blood group 0 negative) and $1250 \mathrm{U}$ factor XIII.

- In the LUKS-Alg, Factor XIII $1250 \mathrm{U}$ (15 U/kg) is considered if FIBTEM maximum clot firmness stays $\leq 7 \mathrm{~mm}$ despite fibrinogen administration, or factor XIII activity is $<60 \%$. According to the USZ-Alg Factor XIII may be administering blindly after $6 \mathrm{~g}$ of fibrinogen or at factor XIII activity $<60 \%$ to improve clot stabilization.

The LUKS-Alg is depicted in detail in the supplemental material (Additional file 1). The USZ-Alg is depicted in detail in the publication of Theusinger et al. [10].

\section{Variables and data collection}

Patient demographics, laboratory values at admission (samples collected within the first minutes after admission to the hospital) and injury epidemiology were extracted from the internal database which is collected and entered by professional medical coders responsible for the data acquisition to the TraumaRegister DGU(R) of the German Trauma Society and copied to a designated spreadsheet (Microsoft ${ }^{\oplus}$ Office Excel 2010, Microsoft ${ }^{\odot}$ Corporation, Redmond, USA). Additionally, the following variables concerning initial resuscitation in the emergency department (hospital admission to ICU admission) were extracted from the anaesthesia records: use of resuscitation fluids, factor concentrates (PCC, factor XIII, recombinant factor VIIa), TXA, vasopressor use (yes/no), duration of treatment and the use of allogeneic blood products such as RBC, FFP and PLT. Multiple cross checks have been performed to ensure high data quality. The median proportion of missing values was $1.4 \%$, IQR 0 to $4.7 \%$.

\section{Endpoints and outcome variables}

The primary endpoints were the comparison between centres / algorithms in the use of allogenic blood products and coagulation factors. Secondary endpoints were the frequency of point of care measurements and haemoglobin level during resuscitation and at admission ICU.

\section{Statistical analyses}

Numerical data reported as mean and standard deviation (SD) or median [Q1;Q3] in the case of skew data distribution and ordinal data (GCS). Categorical data shown in absolute numbers (n) and percent (\%). For the primary analysis, two propensity score matched groups $(n=172$ per group) were computed using a non-parsimonious logistic regression. Skew data was logarithmically transformed (time in the ED, time prehospital, platelet count, 
lactate). Propensity score matching was performed on the logit scale with a caliper of 0.2 standard deviations of the logit of the propensity score. Missing values were replaced by multiple regression imputation for the respective analysis (dataset was generated using 10 iterations of automatic (linear or logistic) regressions depending on the type of the variable). The model fit was assessed using the Hosmer-Lemeshow test. The model was well calibrated (chi-square with 8 degrees of freedom $=11.4, p=0.16$ ). Standardized difference was calculated for the (partially logarithmically transformed) explanatory variables (Table 1) to assess the balance on base line characteristics after propensity score matching. Odds ratios (OR) and 95\% CI were calculated using logistic regression. The Mann-Whitney test was used to compare continuous data between the groups. Statistical significance was set as a two-tailed $p$-value $\leq 0.05$. All statistical analyses were performed by IBM SPSS Statistics 22 (Armonk, NY: IBM Corp).

\section{Results}

A total of 632 patients meeting the inclusion criteria were admitted to the two hospitals: 428 patients were admitted to the USZ and 204 to the LUKS (Fig. 1). Two Propensity score matched groups ( $n=172$ per group) were created with the explanatory variables in Table 1. The absolute standardized difference after matching was $\leq 0.13$ for all explanatory variables, stating an acceptable balance between the matched groups (Table 1).

\section{Usage of blood products, coagulations factors and resuscitation fluids}

Treatment with USZ-Alg compared with LUKS-Alg resulted in a lower number of patients receiving $\mathrm{RBC}$ transfusion (11.6\% vs. $29.7 \%$, OR $3.2,95$ CI $1.8-5.7, p<$ 0.001 , Table 2) and a lower amount of RBC transfusion (0.5 SD 1.9 vs. 1.5 SD 3.9, $p<0.001$, Table 3). Concerning FFP and PLT transfusions, the number of patients transfused and the amount of transfused units did not differ between the algorithms. In patients treated according to the USZ-Alg, mean haemoglobin level in transfused patients was lower during resuscitation (8.0 SD 1.7 vs. 9.4 SD $1.8 \mathrm{~g} / \mathrm{dl}, p<0.001)$ and at ICU admission (8.3 SD 1.2 vs. 10.6 SD $1.9 \mathrm{~g} / \mathrm{dl}, p<0.001$, Figs. 2 and 3).

If treated according to the USZ-Alg, significantly more patients received factor XIII, but less patients received PCC and fibrinogen concentrate (Table 2). The mean overall dose was higher for factor XIII and lower for PCC and fibrinogen if USZ-Alg was applied (Table 3). None of the patients received recombinant factor VIIa.

Table 1 Overview of patient characteristics, mechanisms of injury, management and first laboratory values determined at admission in the propensity score matched groups comparing two 1 trauma centres (LUKS: Cantonal Hospital Lucerne, USZ: University Hospital Zurich)

\begin{tabular}{llll}
\hline & USZ $(n=172)$ & LUKS $(n=172)$ & Standardized difference \\
\hline Age (years) & $53.6(21.8)$ & $51.1(19.7)$ & 0.09 \\
Sex (male) & $119(69 \%)$ & $121(70 \%)$ & 0.03 \\
Heart rate (bpm) on admission & $90(23)(n=169)$ & $91(21)(n=167)$ & 0.04 \\
Systolic blood pressure (mmHg) on admission & $131(27)(n=171)$ & $132(31)(n=168)$ & 0.04 \\
Penetrating trauma & $8(5 \%)$ & $6(4 \%)(n=169)$ & 0.06 \\
GCS on scene & $13[6 ; 15](n=167)$ & $13[6 ; 15](n=169)$ & 0.09 \\
ISS & $27.8(14.5)$ & $27.9(11.5)$ & 0.01 \\
Time (min.) prehospital & $65[55 ; 84]$ & $70[55 ; 90](n=143)$ & $0.01^{\#}$ \\
Time (min.) ED & $145[100 ; 255]$ & $170[105 ; 309](n=143)$ & $0.11^{\#}$ \\
Prehospital intubation & $48(28 \%)$ & $56(33 \%)$ & 0.10 \\
Vasopressor use (in the ED) & $90(52 \%)$ & $93(54 \%)$ & 0.03 \\
First laboratory values determined after admission to the ED & & \\
$\quad$ Haemoglobin (g/dl) & $12.1(2.1)(n=166)$ & $12.1(2.3)(n=169)$ & 0.00 \\
Platelet count (G/l) & $186[152 ; 225](n=167)$ & $197[164 ; 227](n=164)$ & $0.10^{\#}$ \\
Base Excess (mmol/l) & $-3.5(4.9)(n=165)$ & $-2.8(5.5)(n=165)$ & 0.12 \\
Lactate (mmol/) & $1.7[1.1 ; 2.6](n=166)$ & $1.7[1.0 ; 3.0](n=148)$ & $73(19)(n=166)$ \\
Quick's value (\%) & $71(22)(n=167)$ & $2.3(0.8)(n=162)$ & $0.13^{\#}$ \\
Fibrinogen (g/l) & $2.3(1.0)(n=160)$ & 0.09 & 0.07
\end{tabular}

Data reported as frequency $(\mathrm{n})$ with percentage $(\%)$, mean (SD) or median [Q1;Q3]. Standardized difference for the explanatory variables $=$ absolute difference in means or proportions divided by pooled standard deviation. \#: standardized difference computed for logarithmically transformed variable. Standardized difference is $\leq 0.13$ for all explanatory variables, stating an acceptable balance between the matched groups

$B P M$ beats per minute, ED emergency department, GCS glasgow coma scale, ISS injury severity score 
Table 2 Differences in the number of patients receiving allogeneic blood products, coagulation factors and resuscitation fluids (independent of amount) between the propensity score matched groups (LUKS: Cantonal Hospital Lucerne, USZ: University Hospital Zurich)

\begin{tabular}{lllll}
\hline & USZ $(n=172)$ & LUKS $(n=172)$ & OR $(95 \% \mathrm{Cl})$ & $p$-value \\
\hline RBC & $20(11.6 \%)$ & $51(29.7 \%)$ & $3.2(1.8-5.7)$ & $<0.001$ \\
FFP & $4(2.3 \%)$ & $9(5.2 \%)$ & $2.3(0.7-7.7)$ & 0.16 \\
PLT & $10(5.8 \%)$ & $6(3.5 \%)$ & $0.6(0.2-1.6)$ & 0.31 \\
TXA & $76(44.2 \%)$ & $66(28.6 \%)$ & $0.8(0.5-1.2)$ & 0.29 \\
Fibrinogen & $42(24.4 \%)$ & $59(34.3 \%)$ & $1.6(1.0-2.6)$ & 0.04 \\
PCC & $17(9.9 \%)$ & $41(23.8 \%)$ & $2.9(1.5-5.3)$ & 0.001 \\
Factor XIII & $15(8.7 \%)$ & $6(3.5 \%)$ & $0.4(0.1-1.0)$ & 0.04 \\
Starch & $5(2.9 \%)$ & $37(21.5 \%)$ & $9.2(3.5-23.9)$ & $<0.001$ \\
Gelatin & $47(27.3 \%)$ & $27(15.7 \%)$ & $0.5(0.3-0.8)$ & 0.009 \\
\hline
\end{tabular}

Data reported as frequency $(\mathrm{n})$ with percentage (\%). Odds ratio (OR) with $95 \%$ confidence intervals $(\mathrm{Cl})$ and $p$-values were calculated using logistic regression. Level of significance 0.05

FFP fresh frozen plasma, FXIII coagulation factor XIII, PCC 4 factor prothrombin complex concentrate, $P L T$ platelet concentrate, $R B C$ red blood cell concentrate, TXA tranexamic acid

Patients treated according to the USZ algorithm received significantly lower volumes of crystalloids (Table 3), less frequently and lower volumes of hydroxyethyl starch but more frequently and higher volumes of gelatin (Tables 2 and 3).

\section{Point of care measurements during resuscitation}

In patients receiving $\mathrm{RBC}$, more blood gas analyses were made at the USZ (1.4 SD 0.8 vs. 1.0 SD 0.7 measurements per hour, $p=0.004$ ). No significant differences in the amount of ROTEM $^{\circ}$ analyses could be observed

Table 3 Differences in the amount/quantity of administered allogeneic blood products, coagulation factors and resuscitation fluids between the propensity score matched groups (LUKS: Cantonal Hospital Lucerne, USZ: University Hospital Zurich)

\begin{tabular}{llll}
\hline & USZ $(n=172)$ & LUKS $(n=172)$ & $p$-value \\
\hline RBC (U) & $0.5(1.9)$ & $1.5(3.9)$ & $<0.001$ \\
FFP (U) & $0.1(0.6)$ & $0.4(2.2)$ & 0.15 \\
PLT (U) & $0.1(0.3)$ & $0.1(0.4)$ & 0.32 \\
FFP:RBC (ratio) & $0.1(0.3)$ & $0.1(0.3)$ & 0.91 \\
Fibrinogen (g) & $1.1(2.6)$ & $1.5(3.0)$ & 0.05 \\
PCC (IU) & $124(408)$ & $454(1011)$ & $<0.001$ \\
Factor XIII (IU) & $116(388)$ & $51(282)$ & 0.04 \\
Crystalloid (ml) & $2130(2642)$ & $3944(4064)$ & $<0.001$ \\
Starch (ml) & $13(94)$ & $146(319)$ & $<0.001$ \\
Gelatin (ml) & $347(722)$ & $142(392)$ & 0.004 \\
\hline
\end{tabular}

Data reported as mean (SD). The $p$-value (Mann-Whitney test) was calculated between the groups. Level of significance 0.05

FFP fresh frozen plasma, FXIII coagulation factor XIII, PCC 4 factor prothrombin complex concentrate, $P L T$ platelet concentrate, $R B C$ red blood cell concentrate between both algorithms ( 0.5 SD 0.4 vs. 0.5 SD 0.4 measurements per hour, $p=0.83$ ).

\section{Discussion}

The treatment of severely injured patients with individualized goal-directed factor based coagulation and transfusion algorithms is becoming more and more common nowadays and many hospitals already implemented such algorithms. Although common practice, to the best of our knowledge this is the first study investigating the impact of differences in coagulation algorithms on the administration of allogeneic blood products and coagulation factors, point of care measurements and haemoglobin level during resuscitation and at admission ICU.

Our main findings were 1) a target haematocrit-range may lead to less transfusion of $\mathrm{RBC}$ compared to a lower haematocrit limit only, 2) especially in patients requiring allogenic blood products frequently repeated haemoglobin measurement may be crucial to monitor transfusion management.

Following to the USZ-Alg, frequency and amount of $\mathrm{RBC}$ transfusions were significantly lower. In severely injured trauma patients RBC transfusion is only recommended according to evidence based low transfusion triggers [14-17]. As RBC do not contain any coagulation factors RBC transfusions lead to dilution of coagulation factors, which may worsen the bleeding [9]. Therefore, in the USZ-Alg a haematocrit-range as RBC transfusion target is established, avoiding excessive RBC transfusion. This may additionally be reflected by the higher amount of ABGA performed per hour at the USZ to focus on the haematocrit-range in transfused patients compared to the LUKS, where the haemoglobin level is considered to be kept upper a lower haematocrit limit only.

In Switzerland, prehospital tranexamic acid administration was initiated after the CRASH-2 trial was published in 2011 [18]. Therefore, the use of tranexamic acid may be underestimated for both centres, because prehospital administration of tranexamic acid was not analysed. No additional tranexamic acid was given and documented in the ED, if prehospital TXA was already applied. Early empirical administration of tranexamic acid is recommended by the European Trauma Treatment Guidelines to bleeding trauma patients or patients at risk of significant haemorrhage [8].

A difference in fibrinogen concentrate administration was observed, which may be due to goal-directed use according to the USZ-Alg, compared to empirical (partially) use of fibrinogen concentrate according to the LUKS-Alg. As fibrinogen concentrate substitution reduces the need for FFP and RBC transfusion in trauma patients [19], rapid assessment and correction of the initial fibrinogen level is recommended by both algorithms. 


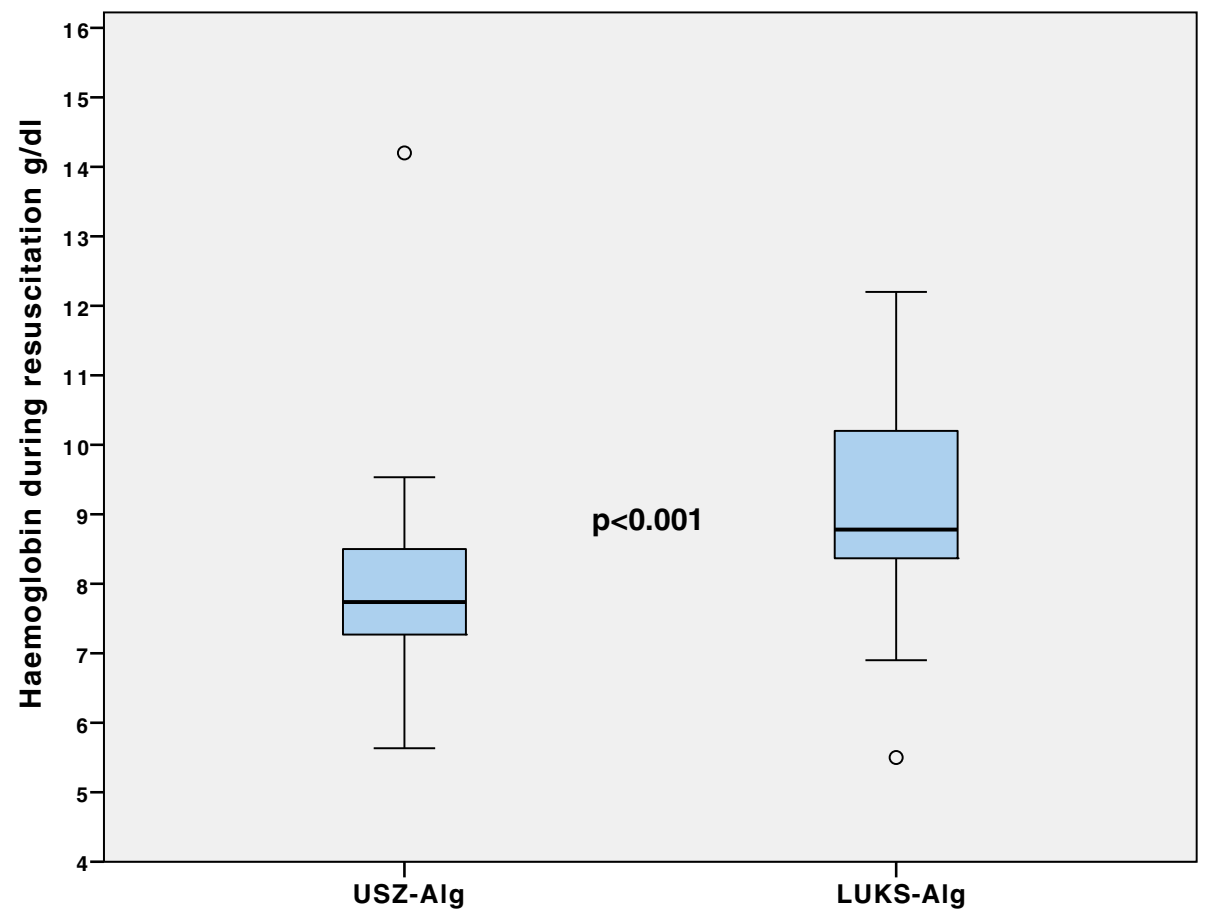

Fig. 2 Mean haemoglobin during resuscitation at the ED. In Patients from the propensity score matched groups requiring at least 1 RBC, mean haemoglobin level during resuscitation at the emergency department was significantly different between both trauma centres: $p<0.001$. RBC: red blood cell concentrate; ED: emergency department; USZ-Alg: coagulation and transfusion algorithm of the University Hospital Zurich; LUKS-Alg: coagulation and transfusion algorithm of the Cantonal Hospital Lucerne

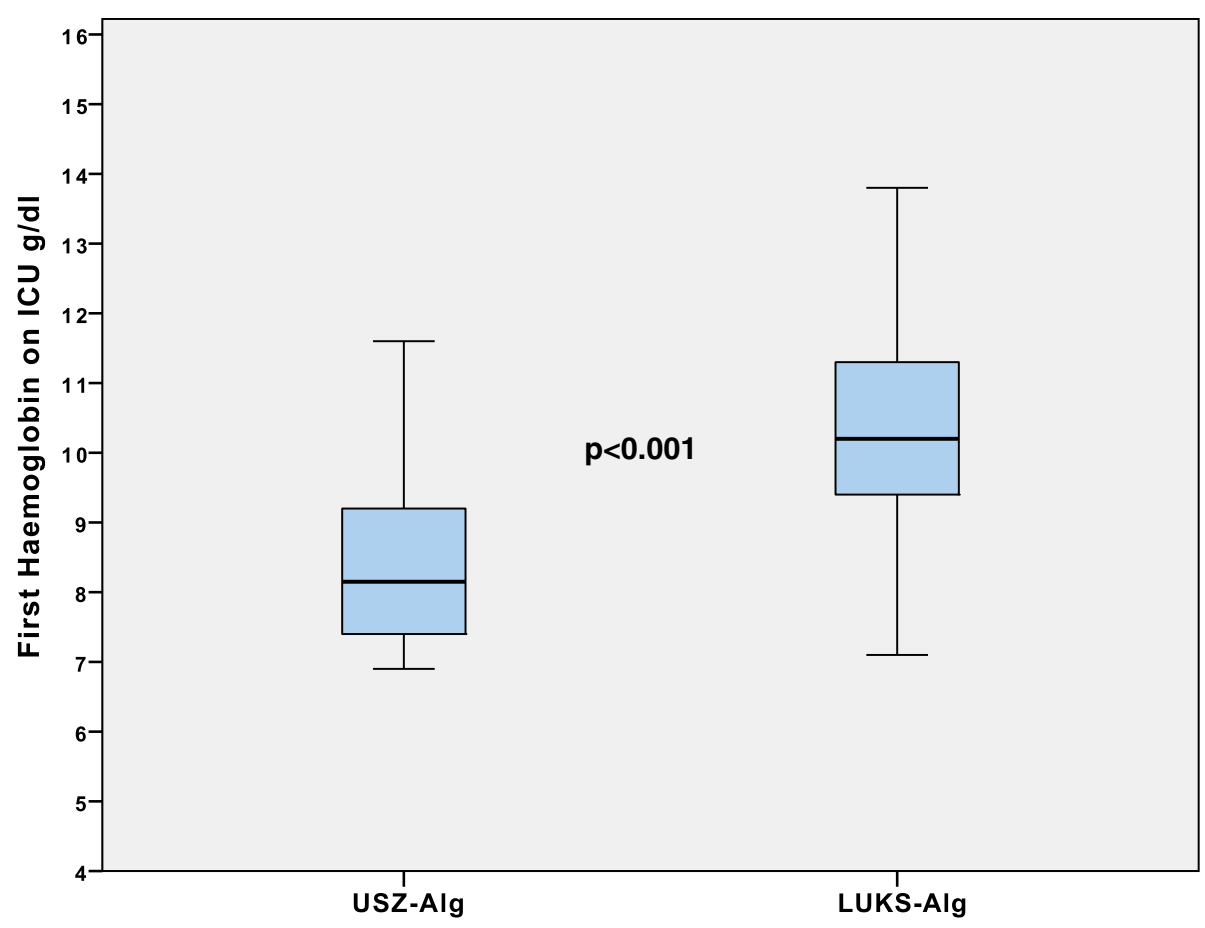

Fig. 3 First haemoglobin on ICU. In patients from the propensity score matched groups requiring at least 1 RBC, first haemoglobin level on admission to the ICU was significantly different between both trauma centres: $p<0.001$. ICU: intensive care unit; RBC: red blood cell concentrate; USZ-Alg: coagulation and transfusion algorithm of the University Hospital Zurich; LUKS-Alg: coagulation and transfusion algorithm of the Cantonal Hospital Lucerne 
At the LUKS patients received PCC more frequently and at a higher dosage. Although PCC is part of the "blind package" for selected trauma cases according to LUKS-Alg, the European Trauma Treatment Guidelines recommend PCC administration only to bleeding patients with documented evidence of delayed coagulation initiation (goal-directed approach) and to trauma patients who are anticoagulated with vitamin $\mathrm{K}$ antagonists [8]. Treating patients blindly with PCC is therefore not recommended and may explain the observed difference for PCC between both centres.

The majority of trauma patients in both centres is no longer exposed to FFP transfusions. As FFP was the only source for coagulation factor XIII, this factor may reach critically low values in bleeding patients with progressive haemodilution. Therefore, measurement and early administration of factor XIII is recommended. It was shown that factor XIII stabilizes the fibrin clot in vitro [20] and reduces postoperative bleeding in cardiac surgery [21]. More coagulation factor XIII was administered at the USZ as it is earlier recommended according to the USZ-Alg.

In both trauma centres, crystalloid solutions are used primarily for fluid resuscitation. Patients at the USZ received a more restrictive volume resuscitation. Restrictive fluid resuscitation is recommended to avoid dilution of coagulation factors, which impairs coagulation additionally until the bleeding is surgically controlled [8]. On the other hand the survival $>=72 \mathrm{~h}$ in severely injured patient is associated with a higher amount of especially saline solutions indicating that some patients may benefit from a more generous fluid resuscitation [22]. The overall numbers of patients that received colloids were comparable between the LUKS and the USZ. Whereas at the USZ used predominantly gelatin, the LUKS used gelatin and hydroxyethyl starch equally frequently throughout the study period, despite the fact both algorithms recommend the use of gelatin. This might be explained by the fact, that the negative, nonreversible effect of starch on coagulation was postulated during the observed study period. It was shown that colloid solutions are affecting coagulation as well as they are an independent predictor of SIRS and Sepsis in severely injured patients [23-25]. Thereby especially starch worsens blood coagulation by impairing fibrin polymerization and platelet function $[20,26]$. In contrast to gelatin, this negative impact on coagulation of hydroxyethyl starch is not reversible by adding coagulation factor concentrates $[23,26]$.

Individualized goal-directed factor concentrate-based algorithms are based on point of care measurements and routine laboratory measurements. Point of care measurements, including viscoelastic testing of the developing clot (e.g. ROTEM ${ }^{\circ}$ ) and ABGA, allow rapid and tailored coagulation and transfusion treatment [3, 27]. The FIBTEM test in viscoelastic testing with ROTEM ${ }^{\circ}$ allows to identify low functional fibrinogen levels within 5-10 min [27]. Low levels are shown to be predictive for massive transfusion in trauma patients [19]. A ROTEM ${ }^{\circ}$-guided haemostatic therapy with fibrinogen concentrate and PCC led to a reduction in the administration of allogeneic blood products in trauma patients [3, 4, 28, 29]. Moreover, a TEG $^{\bullet}$-guided protocol was shown to be superior to a massive transfusion protocol with a fixed ratio strategy in patients with penetrating trauma [2]. It is therefore crucial that point of care measurements are frequently repeated to guide and monitor the coagulation and transfusion management, especially after administering coagulation factors and allogeneic blood products.

\section{Limitations}

Our study has several limitations hat may have interfered with our results. Data analyses were performed retrospectively. However, data quality is high, as all the emergency department data have been collected independently and cross-checked. Moreover, documentation was performed according to the Good Clinical Practice guidelines. Both trauma centres have a standardized approach for the initial treatment of severely injured patients based on to leading trauma guidelines, such as ATLS $^{\circ}$ or ETC. Nevertheless, apart from the differences in the coagulation algorithms, also other differences in the treatment of trauma patients may not be accounted for despite the fact that after matching, demographic and trauma epidemiology including admission laboratory values did not differ between the groups. From this study design, therefore only associations but no causal relationships may be derived. Treatment differences may not be limited to the investigated algorithms only, but also to undetermined differences between the study centres. Conducting a prospective multicentre study to investigate 2 coagulation algorithms in parallel would be difficult to perform, as for example blinding is not possible. Therefore, comparing two coagulation algorithms retrospectively and showing differences in the transfusion of allogeneic blood products may be considered a pragmatic approach.

\section{Conclusion}

A goal-directed coagulation algorithm including a target haematocrit-range including frequent and repeated haematocrit measurement may lead to less transfusion of RBC compared to only a lower haematocrit limit, when treating severely traumatized patients. 


\section{Additional file}

Additional file 1: Transfusion and coagulation algorithm of the Cantonal Hospital Lucerne (LUKS-Alg). (PDF 23 kb)

\section{Abbreviations}

ABGA: Arterial blood gas analysis; aPTT: Activated partial thromboplastin time; BE: Base excess; Cl: Confidence interval; ED: Emergency department; FFP: Fresh frozen plasma; GCS: Glasgow coma scale; ICU: Intensive care unit INR: International normalized ratio; ISS: Injury severity score; LUKS: Cantonal Hospital Lucerne; LUKS-Alg: Transfusion and coagulation algorithm of the Cantonal Hospital Lucerne; OR: Odds ratio; PCC: Prothrombin complex concentrate; PLT: Platelet concentrate; RBC: Red Blood Cell concentrate; ROTEM: Rotational Thomboelastometry; SD: Standard deviation; TXA: Tranexamic acid; USZ: University Hospital Zurich; USZ-Alg: Transfusion and coagulation algorithm of the University Hospital Zurich

\section{Acknowledgements}

Not applicable.

\section{Funding}

The study was not funded.

\section{Availability of data and materials}

The datasets used and/or analysed during the current study available from the corresponding author on reasonable request. Standard software programs (SPSS, Excel, Word, Endnote) were used to complete this study. They are available to any scientists. No new tools were used to analyse this study.

\section{Authors' contributions}

AK and PS participated in the design and coordination of the study, contributed to data collection, statistical analysis, data interpretation, drafting the manuscript and critical revision of the manuscript. BS contributed to statistical analysis, data interpretation and critical revision of the manuscript. DS, MC, KS and RM contributed to data interpretation and critical revision of the manuscript. All authors read and approved the final manuscript.

\section{Ethics approval and consent to participate}

This study was approved by the local ethics committee (Kantonale Ethikkommission Zürich, Switzerland, KEK ZH 2015-0309;). An individual patient consent was waived by the ethics committee in the context of a large retrospective study. All necessary written informed consent from any patients involved in the study, including consent to participate in the study was obtained.

\section{Consent for publication}

Not applicable.

\section{Competing interests}

AK, MC, KS, BS have no conflicts of interest. PS received honoraria for lecturing by Vifor Pharma (Munich, Germany). Donat R. Spahn's academic department is receiving grant support from the Swiss National Science Foundation, Berne, Switzerland, the Ministry of Health (Gesundheitsdirektion) of the Canton of Zurich, Switzerland for Highly Specialized Medicine, the Swiss Society of Anesthesiology and Reanimation (SGAR), Berne, Switzerland, the Swiss Foundation for Anesthesia Research, Zurich, Switzerland, Bundesprogramm Chancengleichheit, Berne, Switzerland, CSL Behring, Berne, Switzerland, Vifor SA, Villars-sur-Glâne, Switzerland. Dr. Spahn was the chair of the $A B C$ Faculty and is the co-chair of the ABC-Trauma Faculty, which both are managed by Physicians World Europe $\mathrm{GmbH}$, Mannheim, Germany and sponsored by unrestricted educational grants from Novo Nordisk Health Care AG, Zurich, Switzerland, CSL Behring GmbH, Marburg, Germany and LFB Biomédicaments, Courtaboeuf Cedex, France. In the past 5 years, Dr. Spahn has received honoraria or travel support for consulting or lecturing from the following companies and organizations: Danube University of Krems, Austria; US Department of Defense, Washington, USA; European Society of Anesthesiology, Brussels, BE; Baxter AG, Volketswil, Switzerland, Baxter S.p.A., Roma, Italy;Bayer (Schweiz) AG, Zürich, Switzerland; Bayer Pharma AG, Berlin, Germany; B. Braun Melsungen AG, Melsungen, Germany; Boehringer Ingelheim (Schweiz) GmbH, Basel, Switzerland; Bristol-Myers-Squibb, Rueil-
Malmaison Cedex, France and Baar, Switzerland; CSL Behring GmbH, Hattersheim am Main, Germany and Berne, Switzerland; Curacyte AG, Munich, Germany; Daiichi Sankyo (Schweiz) AG, Thalwil, Switzerland; Ethicon Biosurgery, Sommerville, New Jersey, USA; Fresenius SE, Bad Homburg v.d.H., Germany; Galenica AG, Bern, Switzerland (including Vifor SA, Villars-sur-Glâne, Switzerland); GlaxoSmithKline GmbH \& Co. KG, Hamburg, Germany; Haemonetics, Braintree, MA, USA; Janssen-Cilag AG, Baar, Switzerland, Janssen-Cilag EMEA, Beerse, Belgium; LFB Biomédicaments, Courtaboeuf Cedex, France; Merck Sharp \& Dohme AG, Luzern, Switzerland; Novo Nordisk A/S, Bagsvärd, Denmark; Octapharma AG, Lachen, Switzerland; Oxygen Biotherapeutics, Costa Mesa, CA; PAION Deutschland GmbH, Aachen, Germany; Pharmacosmos A/S, Holbaek, Denmark; Photonics Healthcare B.V., Utrecht, Netherlands; ratiopharm Arzneimittel Vertriebs-GmbH, Vienna, Austria; Roche Diagnostics International Ltd., Reinach, Switzerland; Roche Pharma (Schweiz) AG, Reinach, Switzerland; Sarstedt AG \& Co., Sevelen, Switzerland and Nümbrecht, Germany; Schering-Plough International, Inc., Kenilworth, New Jersey, USA; Tem International GmbH, Munich, Germany; Verum Diagnostica GmbH, Munich, Germany; Vifor Pharma Deutschland $\mathrm{GmbH}$, Munich, Germany; Vifor Pharma Österreich GmbH, Vienna, Austria; Vifor (International) AG, St. Gallen, Switzerland.

\section{Publisher's Note}

Springer Nature remains neutral with regard to jurisdictional claims in published maps and institutional affiliations.

\section{Author details}

${ }^{1}$ Institute of Anaesthesiology, University and University Hospital Zürich, Raemistrasse 100, 8091 Zürich, Switzerland. ²Department of Anaesthesiology and Intensive Care, Cantonal Hospital Lucerne, Spitalstrasse 16, 6000 Luzern, Switzerland. ${ }^{3}$ Department of Traumatology, University and University Hospital Zürich, Raemistrasse 100, 8091 Zürich, Switzerland. ${ }^{4}$ Epidemiology, Biostatistics and Prevention Institute, Department of Biostatistics, University of Zurich, Hirschengraben 84, 8001 Zurich, Switzerland

Received: 25 July 2017 Accepted: 28 November 2017 Published online: 08 January 2018

\section{References}

1. Stein P, Kaserer A, Sprengel K, Wanner GA, Seifert B, Theusinger OM, et al. Change of transfusion and treatment paradigm in major trauma patients. Anaesthesia. 2017;72(11):1317-1326

2. Tapia NM, Chang A, Norman M, Welsh F, Scott B, Wall MJ Jr, et al. TEG-guided resuscitation is superior to standardized MTP resuscitation in massively transfused penetrating trauma patients. J Trauma Acute Care Surg. 2013;74(2):378-85. discussion 385-6

3. Schöchl H, Nienaber U, Maegele M, Hochleitner G, Primavesi F, Steitz B, et al. Transfusion in trauma: thromboelastometry-guided coagulation factor concentrate-based therapy versus standard fresh frozen plasma-based therapy. Crit Care. 2011;15(2):R83.

4. Gonzalez E, Moore EE, Moore HB, Chapman MP, Chin TL, Ghasabyan A, et al. Goal-directed hemostatic resuscitation of trauma-induced coagulopathy: a pragmatic randomized clinical trial comparing a viscoelastic assay to conventional coagulation assays. Ann Surg. 2016;263(6):1051-9.

5. Theusinger OM, Wanner GA, Emmert MY, Billeter A, Eismon J, Seifert B, et al. Hyperfibrinolysis diagnosed by rotational Thromboelastometry $\left(\mathrm{ROTEM}^{\circledR}\right)$ is associated with higher mortality in patients with severe trauma. Anesth Analg. 2011:113(5):1003-12.

6. Gando S, Hayakawa M. Pathophysiology of trauma-induced coagulopathy and management of critical bleeding requiring massive transfusion. Semin Thromb Hemost. 2016;42(2):155-65.

7. Vogt KN, Van Koughnett JA, Dubois L, Gray DK, Parry NG. The use of trauma transfusion pathways for blood component transfusion in the civilian population: a systematic review and meta-analysis. Transfus Med. 2012;22(3):156-66.

8. Rossaint R, Bouillon B, Cerny V, Coats TJ, Duranteau J, Fernández-Mondéjar $E$, et al. The European guideline on management of major bleeding and coagulopathy following trauma: fourth edition. Crit Care. 2016:20(1):100.

9. Theusinger OM, Spahn DR, Ganter MT. Transfusion in trauma: why and how should we change our current practice? Curr Opin Anaesthesiol. 2009;22(2):305-12 
10. Theusinger OM, Madjdpour C, Spahn DR. Resuscitation and transfusion management in trauma patients: emerging concepts. Curr Opin Crit Care. 2012;18(6):661-70.

11. Theusinger OM, Stein $P$, Spahn DR. Applying 'Patient blood Management' in the trauma center. Curr Opin Anaesthesiol. 2014;27(2):225-32.

12. von Elm E, Altman DG, Egger M, Pocock SJ, Gotzsche PC, Vandenbroucke $J P$, et al. The strengthening the reporting of observational studies in epidemiology (STROBE) statement: guidelines for reporting observational studies. Bull World Health Organ. 2007;85(11):867-72.

13. Gordic S, Alkadhi H, Hodel S, Simmen HP, Brueesch M, Frauenfelder T, et al. Whole-body CT-based imaging algorithm for multiple trauma patients: radiation dose and time to diagnosis. Br J Radiol. 2015;88(1047):20140616.

14. Spahn DR. Coagulopathy and blood component transfusion in trauma. $\mathrm{Br}$ J Anaesth. 2005;95(2):130-9.

15. Spahn DR, Spahn GH, Stein P. Evidence base for restrictive transfusion triggers in high-risk patients. Transfus Med Hemother. 2015;42(2):110-4.

16. Theusinger OM, Stein P, Spahn DR. Transfusion strategy in multiple trauma patients. Curr Opin Crit Care. 2014;20(6):646-55.

17. Desborough MJ, Colman KS, Prick BW, Duvekot JJ, Sweeney C, Odutayo A, et al. Effect of restrictive versus liberal red cell transfusion strategies on haemostasis: systematic review and meta-analysis. Thromb Haemost. 2017;117(5):889-898.

18. CRASH-2 trial collaborators, Shakur H, Roberts I, Bautista R, Caballero J, Coats $T$, et al. Effects of tranexamic acid on death, vascular occlusive events, and blood transfusion in trauma patients with significant haemorrhage (CRASH-2): a randomised, placebo-controlled trial. Lancet. 2010;376(9734):23-32.

19. Schochl H, Cotton B, Inaba K, Nienaber U, Fischer H, Voelckel W, et al. FIBTEM provides early prediction of massive transfusion in trauma. Crit Care. 2011;15(6):R265

20. Theusinger OM, Baulig W, Asmis LM, Seifert B, Spahn DR. In vitro factor XIII supplementation increases clot firmness in rotation Thromboelastometry $\left(\right.$ ROTEM $\left.^{\circledast}\right)$. Thromb Haemost. 2010;104(2):385-91.

21. Gödje O, Gallmeier U, Schelian M, Grünewald M, Mair H. Coagulation factor XIII reduces postoperative bleeding after coronary surgery with extracorporeal circulation. Thorac Cardiovasc Surg. 2006;54(1):26-33.

22. Sprengel K, Simmen H, Werner CML, Jensen $\mathrm{KO}$, Keller $\mathrm{C}$, Wirth $\mathrm{SH}$, et al. Analysis of fluid resuscitation in critically injured patients-a central role of saline solutions. J Acute Med. 2016:6(2):29-34.

23. Kind SL, Spahn-Nett GH, Emmert MY, Eismon J, Seifert B, Spahn DR, et al. Is dilutional coagulopathy induced by different colloids reversible by replacement of fibrinogen and factor XIII concentrates? Anesth Analg. 2013;117(5):1063-71.

24. Skhirtladze K, Base EM, Lassnigg A, Kaider A, Linke S, Dworschak M, et al. Comparison of the effects of albumin 5\%, hydroxyethyl starch 130/0.4 6\%, and Ringer's lactate on blood loss and coagulation after cardiac surgery. $\mathrm{Br} J$ Anaesth. 2014;112(2):255-64.

25. Mica L, Simmen H, Werner CM, Plecko M, Keller C, Wirth SH, et al. Fresh frozen plasma is permissive for systemic inflammatory response syndrome, infection, and sepsis in multiple-injured patients. Am J Emerg Med. 2016;34(8):1480-5.

26. Thaler U, Deusch E, Kozek-Langenecker SA. In vitro effects of gelatin solutions on platelet function: a comparison with hydroxyethyl starch solutions. Anaesthesia. 2005;60(6):554-9.

27. Stein P, Kaserer A, Spahn GH, Spahn DR. Point-of-care coagulation monitoring in trauma patients. Semin Thromb Hemost. 2017;43(4):367-74.

28. Tauber H, Innerhofer P, Breitkopf R, Westermann I, Beer R, El Attal R, et al. Prevalence and impact of abnormal ROTEM(R) assays in severe blunt trauma: results of the 'Diagnosis and treatment of trauma-induced Coagulopathy (DIA-TRE-TIC) study'. Br J Anaesth. 2011;107(3):378-87.

29. Da Luz LT, Nascimento B, Shankarakutty AK, Rizoli S, Adhikari NK. Effect of thromboelastography $(T E G(R))$ and rotational thromboelastometry (ROTEM(R) on diagnosis of coagulopathy, transfusion guidance and mortality in trauma: descriptive systematic review. Crit Care. 2014;18(5):518.

\section{Submit your next manuscript to BioMed Central and we will help you at every step:}

- We accept pre-submission inquiries

- Our selector tool helps you to find the most relevant journal

- We provide round the clock customer support

- Convenient online submission

- Thorough peer review

- Inclusion in PubMed and all major indexing services

- Maximum visibility for your research

Submit your manuscript at www.biomedcentral.com/submit
) Biomed Central 